\title{
Combining Telemetry and Fisheries Tagging Models to Estimate Fishing and Natural Mortality Rates
}

\author{
Kenneth H. PollocK* \\ Departments of Zoology and Statistics, North Carolina State University, \\ Raleigh, North Carolina 27695-7617, USA
}

HONGHUA JIANG

Biomathematics Program, Department of Statistics, North Carolina State University, Raleigh, North Carolina 27695-8203, USA

Joseph E. Hightower

\begin{abstract}
U.S. Geological Survey, North Carolina Cooperative Fish and Wildlife Research Unit, Department of Zoology, North Carolina State University, Raleigh, North Carolina 27695-7617, USA
\end{abstract}

\begin{abstract}
The traditional multiple-year tag return method is a fundamental approach to estimating fishing and natural mortality rates in fisheries. It can provide reliable and precise estimation of the parameters when the tag reporting rate, $\lambda$, is known. However, it is often difficult to estimate $\lambda$ accurately. An additional disadvantage is that this method has to estimate natural mortality indirectly because natural deaths are not observable. Recently, fisheries biologists have begun to implement telemetry methods to estimate fishing and natural mortality. The advantage of a telemetry method is that it contains direct information about natural mortality and survival. A shortcoming is that there is no direct information on fishing deaths. In this paper, we combine the two methods, using direct information about both fishing and natural mortality to obtain more precise and effectively unbiased parameter estimates, including reporting rate estimates for the regular tags. Using simulation when the telemetry tag relocation probability equals 1 , we found that the relative standard error (RSE) of natural mortality estimates in the combination method is much improved relative to those of the tag return and telemetry methods. Annual fishing mortality estimates can also be estimated precisely. As expected, as the relocation probability of telemetry tags decreases the RSE of natural mortality and the other parameter estimates increases, but only slightly. Studies clearly should have relocation probabilities that are as high as possible. The tag reporting rate estimates are typically precisely estimated in the combined model. This is very important, as the tag reporting rate is not easy to estimate with other methods.
\end{abstract}

Information about the natural mortality rate (in combination with the fishing mortality rate) is necessary for effective fisheries management; however, the natural mortality rate is difficult to estimate directly because of the lack of direct information about natural deaths. There are several methods for estimating the instantaneous natural mortality rate ( $M$; Hightower et al. 2001): (1) catch curve analysis, (2) regression of the instantaneous total mortality rate $(Z)$ on fishing effort, (3) correlation of $M$ with other life history parameters (Vetter 1988; Quinn and Deriso 1999), (4) tag return methods, and (5) telemetry methods (Hightower et al. 2001). Only $Z$ can be estimated from catch curve analyses. For unfished populations, catch curve analysis can provide an estimate of $M$ because $Z$ and $M$ are the same in this case. A re-

\footnotetext{
* Corresponding author: pollock@unity.ncsu.edu

Received February 7, 2003; accepted October 30, 2003
}

gression equation using $Z$ and fishing effort (Ricker 1975) to estimate $M$ can be used with exploited populations. However, it is often difficult to estimate fishing effort and $Z$ reliably. The advantage of relating $M$ to life history parameters, such as maximum age or growth rate, is that it requires a minimal amount of data (Vetter 1988), but the estimates obtained are of unknown precision (Vetter 1988; Pascual and Iribarne 1993). Tag return methods have also been used to estimate natural and fishing mortality rates (e.g., Pollock et al. 1991; Hoenig et al. 1998a, 1998b). Using telemetry methods is a fairly new approach to estimating natural and fishing mortality rates. Here we discuss the last two methods in detail.

The use of tag return methods to estimate fishing and natural mortality rates has recently received attention in fisheries research. One drawback of this method is that the tag reporting rate is needed, and that is difficult to estimate. Pollock et al. (1991) show that one can determine the instanta- 
neous rates of fishing and natural mortality if the tag reporting rate is estimated either by conducting a reward tagging study or by means of a creel survey or port sampling. Hoenig et al. (1998a) incorporated fishing effort into multiyear tag return studies to estimate the natural mortality rate and the catchability coefficient under the assumption that the tag reporting and retention-survival rates (the short-term probability of retaining the tag and surviving the tagging process) were known. They also estimated the catchability coefficient, the natural mortality rate, and the product of the tag reporting and retention-survival rates for cases in which the reporting and retentionsurvival rates were not known. Although they could estimate tag reporting rates with multiyear tag return data, they found that the estimates were not stable. One limitation of multiyear tag return studies is that they require the assumption that the newly tagged animals are completely mixed with and have the same survival rates as the previously tagged ones after release. Hoenig et al. (1998b) built models that allow for incomplete mixing of newly tagged animals. Pollock et al. (1991), and more recently Pollock et al. $(2001,2002)$ and Hearn et al. (2003), have made a systematic study of methods of estimating the tag reporting rate that are external to the tagging data (high-reward tagging, planted tags, observers, etc.). Hearn et al. (1998) developed pre- and postseason tagging models that allow one to estimate reporting rates and fishing and natural mortality rates from tag return data alone.

Telemetry methods are becoming increasingly popular ways to estimate mortality rates for terrestrial animals. Pollock et al. (1989a, 1989b) used the Kaplan-Meier method (Kaplan and Meier 1958), and later Pollock et al. (1995) developed a more general approach that allows for uncertain relocation by linking the Jolly-Seber open capture-recapture method (Seber 1982) and the Kaplan-Meier method. The key advantage of the telemetry approach is that information about live animals and the natural mortality (nonharvest) of animals can be obtained at shorter time intervals (we usually only obtain annual information about fishing mortality in multiyear tag return studies). Realizing the valuable advance of the telemetry mortality methods, Hightower et al. (2001) first applied them to fish populations by estimating the natural and fishing mortality rates of striped bass Morone saxatilis stocked into Lake Gaston, North Carolina-Virginia.

Recently, biologists have started to use joint
TABLE 1.- Representative matrix of observed tag recoveries in the tag return model.

\begin{tabular}{ccccccc}
\hline & \multirow{6}{*}{$\begin{array}{c}\text { Number } \\
\text { Year }\end{array}$} & \multicolumn{5}{c}{ Year recovered } \\
\cline { 2 - 7 } & tagged & 1 & 2 & 3 & $\cdots$ & $I$ \\
\hline 1 & $N_{1}$ & $R_{11}$ & $R_{12}$ & $R_{13}$ & $\cdots$ & $R_{1 I}$ \\
2 & $N_{2}$ & & $R_{22}$ & $R_{23}$ & $\cdots$ & $R_{2 I}$ \\
3 & $N_{3}$ & & & $R_{33}$ & $\cdots$ & $R_{3 I}$ \\
$\vdots$ & $\vdots$ & & & & $\cdots$ & $\vdots$ \\
$I$ & $N_{I}$ & & & & & $R_{I I}$ \\
\hline
\end{tabular}

analyses of different types of data to estimate survival rates in wildlife studies (Powell et al. 2000; Nasution et al. 2001, 2002). The key advantage of these combined methods is that they make full use of the available information about animals that are alive, harvested, and naturally dead and therefore provide more precise - and relatively unbiasedestimates. Combining tag return and telemetry methods in fish populations is a promising approach for obtaining more precise and relatively unbiased estimates of natural and fishing mortality rates. Another advantage of the combined method is that one can use it to estimate the tag reporting rate. We develop a model structure to estimate natural and fishing mortality rates and tag reporting rates by combining tag return and telemetry data. We then review the assumptions that are being made. We also use simulations to compare the precision of parameter estimates in combined analyses with that in separate analyses. We conclude with a general discussion and suggestions for future research.

\section{Model Development}

\section{Fisheries Tag Return Models}

Fisheries tag return models were used to estimate natural and fishing mortality rates and tag reporting rates (Pollock et al. 1991; Hearn et al. 1998; Hoenig et al. 1998a, 1998b). Here we consider the typical once-a-year tagging at the beginning of each year for multiple years with annual tag returns.

Let $N_{i}$ be the number of fish tagged and released in year $i$ for $i=1,2, \ldots, I$.

Let $R_{i j}$ be the number of fish tagged in year $i$ and exploited and reported in year $j$ for $j=i, i+$ $1, i+2, \ldots, I$.

The observed tag recovery data can be arrayed in a matrix of the form shown in Table 1.

The expected number of fish tagged in year $i$ and recaptured and returned in year $j$ is 


$$
E\left[R_{i j}\right]=N_{i} \cdot P_{i j}
$$

where

$P_{i j}= \begin{cases}\left(\prod_{k=i}^{j-1} S_{k}\right)\left(1-S_{j}\right)\left(\frac{F_{j}}{F_{j}+M}\right) \lambda & (\text { when } j \neq i) \\ \left(1-S_{j}\right) \cdot F_{j} /\left(F_{j}+M\right) \cdot \lambda & (\text { when } j=i)\end{cases}$

$S_{j}=\exp \left(-F_{j}-M\right)$;

$P_{i j}=$ the probability of fish tagged in year $i$ being recaptured and returned in year $j$

$S_{j}=$ the annual survival rate in year $j$

$F_{j}=$ the instantaneous fishing mortality rate in year $j$, which can change from year to year

$M=$ the instantaneous natural mortality rate, which is assumed constant over time

$\lambda=$ the tag reporting rate (i.e., the probability that the tag will be reported given that a tagged fish is caught).

The number of fish tagged in year $i$ and harvested and returned in year $j$ follows the multinomial distribution. Therefore, the likelihood function is a product multinomial following Hoenig et al. (1998a):

$$
\begin{aligned}
& L_{1}=\prod_{i=1}^{I}\left(\begin{array}{c}
N_{i} \\
R_{i i}, R_{i i+1}, \ldots, R_{i I}
\end{array}\right) \\
& \times \prod_{j=i}^{I}\left[P_{i j}^{R_{i j}}\left(1-\sum_{j=i}^{I} P_{i j}\right)^{N_{i}-\Sigma_{j=i}^{I} R_{i j}}\right] .
\end{aligned}
$$

We can obtain maximum likelihood estimators (MLEs) for $F_{j}$ and $M$ if $\lambda$ is known, or we can estimate $F_{j}, M$, and $\lambda$ if $\lambda$ is unknown.

\section{Telemetry Tagging Models}

Pollock et al. (1995) first developed a general capture-recapture model that allows for the probability $(\leq 1.0)$ that telemetered animals will be relocated. Hightower et al. (2001) extended that model and applied it to fish populations. We used the telemetry method to estimate natural and fishing mortality rates and relocation rates as discussed in detail by Hightower et al. (2001). Suppose that we use $I$ years of telemetered releases and that $R_{i}$ is the number of fish telemetered at the beginning of year $i$ for $i=1,2, \ldots, I$. (Note that we are assuming that the regular and the telemetry tag releases are randomly chosen so as to assure mixing of both tag types throughout the population.) We relocate the telemetered fish monthly. If a fish is first relocated alive (i.e., movement was detectable between searches) at period $j$ for $j=1$, $2, \ldots, 12 I-1$, it becomes part of a new (virtual) release at time $j+1$. Let $N_{j}$ be the new (virtual) release at period $j$ and $n_{j}$ the number of fish first relocated alive at period $j$. Then

$$
N_{j+1}= \begin{cases}n_{j}+R_{j} & (j=12 i, i=1,2, \ldots, I) \\ n_{j} & (j \neq 12 i ; i=1,2, \ldots, I) .\end{cases}
$$

The fish were considered to have suffered a natural death if they were found at the same site on consecutive searches. Consider the first relocation of each telemetered fish in release $N_{j}$. The expected number of fish from the $j$-period new (virtual) release $N_{j}$ first relocated alive at period $j+1$ is

$$
E\left[n_{j+1}\right]=N_{j} \cdot P_{1(j+1)},
$$

where $P_{1(j+1)}$, the probability of fish from $j$-period new (virtual) release $N_{j}$ first being relocated alive at period $j+1$, is as follows:

$$
P_{1(j+1)}=\exp \left(-F_{j}-M\right) \times p_{j+1},
$$

$p_{j+1}$ being the relocation probability at period $j+1$.

The expected number of fish from release $N_{j}$ first relocated alive at period $j+2$ is

$$
E\left[n_{j+2}\right]=N_{j} \cdot P_{1(j+2)},
$$

where

$$
\begin{aligned}
P_{1(j+2)}= & \exp \left(-F_{j}-M\right)\left(1-p_{j+1}\right) \\
& \times \exp \left(-F_{j+1}-M\right) \cdot p_{j+2} .
\end{aligned}
$$

The expected number of fish from release $N_{j}$ first relocated dead at period $j+1$ is

$$
E\left[d_{j+1}\right]=N_{j} \cdot P_{d(j+1)},
$$

where $P_{d(j+1)}$, the probability of fish from release $N_{j}$ first being relocated dead (naturally) at period $j+1$, is as follows:

$$
P_{d(j+1)}=\left[1-\exp \left(-F_{j}-M\right)\right]\left[M /\left(F_{j}+M\right)\right] p_{j+1} .
$$

The expected number of fish from release $N_{j}$ first relocated dead at period $j+2$ is

$$
E\left[d_{j+2}\right]=N_{j} \cdot P_{d(j+2)},
$$

where

$$
\begin{aligned}
P_{d(j+2)}= & \exp \left(-F_{j}-M\right)\left(1-p_{j+1}\right) \\
& \times\left[1-\exp \left(-F_{j+1}-M\right)\right] \\
& \times\left[M /\left(F_{j+1}+M\right)\right] p_{j+2} .
\end{aligned}
$$

We are assuming here that natural deaths occur in the period prior to the fish's being located. The 
likelihood function is again a product of multiple multinomial distributions, where we condition on the $N_{j}$ :

$$
\begin{aligned}
L_{2}= & \prod_{j=1}^{12 I}\left(\begin{array}{c}
N_{j} \\
n_{j+1}, d_{j+1}, n_{j+2}, d_{j+2}, \ldots
\end{array}\right) \\
& \times\left[P_{l(j+1)}^{n_{j+1}} P_{d(j+1)}^{d_{j+1}} P_{l(j+2)}^{n_{j+2}} P_{d(j+2)}^{d_{j+2}} \cdots\right] \\
& \times\left[1-P_{l(j+1)}-P_{d(j+1)}-P_{l(j+2)}\right. \\
& \left.\quad-P_{d(j+2)}-\cdots\right]^{N_{j}-n_{j+1}-d_{j+1}-n_{j+2}-d_{j+2} \cdots}
\end{aligned}
$$

(the three dots indicate that there are additional terms that are not shown). In the special case in which the relocation probability is 1 , the likelihood function simplifies to one multinomial distribution for each telemetered cohort because we know exactly how many natural and fishing deaths there were on each occasion.

\section{A New Combination Model}

We use joint analysis of tag return and telemetry data in the combination model. The likelihood function in this model $(L)$ is the product of the likelihood function of tag return data $\left(L_{1}\right)$ and that of the telemetry data $\left(L_{2}\right)$ because (as we discussed above) the two sets of data are independent:

$$
L=L_{1} \times L_{2} .
$$

In a typical once-a-year tagging return analysis, it is difficult to estimate the tag reporting rate. With the combination method, we have enough information to estimate the tag reporting rate in addition to the fishing and natural mortality rates. Further, if the tag reporting rate is known, we can obtain even more precise estimates of the natural and fishing mortality rates.

\section{Assumptions}

The assumptions underlying the tag return model were discussed in detail by Pollock et al. (1991); those underlying the telemetry model were discussed in detail by Pollock et al. (1995) and Hightower et al. (2001). Here we summarize the assumptions for the combination model.

\section{Assumptions for Tag Return Data}

(1) The tagged sample is representative of the target population or the tagged animals are mixed thoroughly with the untagged ones.

Ideally, the marking study would be designed to let the tagged fish disperse widely over the study areas before fishing starts, thus avoiding their being under heavy fishing pressure immediately after tagging (the same is true for telemetered fish).
(2) There is no tag loss.

When tag loss occurs, fishing mortality rate estimates will be negatively biased. Double tagging can be used to adjust for tag loss (Seber 1982).

(3) Survival rates are not affected by tagging.

If the marking substantially decreases survival, the survival estimates will be negatively biased and the mortality estimates positively biased.

(4) The fate of each tagged fish is independent of the fate of other tagged fish.

This assumption is probably violated in almost all practical applications of tag return models. Fish are not independent entities in terms of survival or other characteristics. This does not cause bias, but it does make the precision lower than it appears (Pollock and Raveling 1982).

(5) The year (fishing season) of tag recovery is correctly tabulated.

If anglers report tags from fish caught in previous years, the survival estimates will be positively biased.

(6) All tagged fish within an identifiable class have the same annual survival and recovery probabilities.

In practice, survival and recovery rates are likely to be heterogeneous. Nichols et al. (1982) and Pollock and Raveling (1982) found that if only the recovery rates are heterogeneous there will be no bias in the survival estimates and the recovery rate estimates can be viewed as averages for the population. If the survival probabilities are also heterogeneous, the survival rate estimators may be positively or negatively biased.

\section{Assumptions for the Telemetry Data}

(1) All marked fish present in the study area at time $i$ (whether alive or dead of natural causes) have the same probability $\left(p_{i}\right)$ of being relocated.

We assume that animals that are alive or dead from a natural cause at a particular sampling time have the same probability of relocation. This may be reasonable in some applications, but in some instances telemetered animals that are alive and moving about may be more easily located than dead ones.

(2) All marked fish alive in the study area at time $i$ have the same survival rate to the next time.

We assume that survival rates are homogeneous. However, there may be situations in which newly marked fish have different survival rates from previously marked ones due to differences in age or other factors.

(3) The probability of transmitter failure or of a transmitter's being shed is negligible. 
This assumption seems reasonable if we apply modern telemetry tags. Also, one could be conservative and truncate all telemetry-tagged fish after a fixed period if this were of concern.

(4) Marked and unmarked fish have the same survival rates.

We assume that the telemetry tag has no effect on survival and that the marked fish are representative of the whole population. Violation of this assumption will negatively bias survival.

(5) All fish behave independently with respect to capture and survival.

This is the same assumption as for the tag return data.

(6) Movement patterns can be used to determine whether a tagged fish remains alive or has died due to natural causes (possibly including hooking mortality).

For example, Hightower et al. (2001) assumed that fish that were located repeatedly at the same site had died of natural or hooking mortality. Ideally, we would like to assume that $M$ is an estimate of natural mortality alone, but sometimes hooking mortality can be substantial (e.g., Muoneke and Childress 1994; Wilde 1998; Wilde et al. 2000).

(7) Natural mortality occurs immediately prior to the first relocation.

It is impossible to determine the timing of natural deaths when a fish is found dead after not being located on one or more occasions.

(8) There is no emigration out of the study area.

We did not consider emigration in our study. If there is emigration, the fishing mortality rate estimates will be positively biased (the apparent $F$ will equal the true $F$ plus the emigration rate in that case). In open systems, such as an estuary or section of river where emigration is possible, automated monitors can often be used to detect movement out of the study area so that emigrants can be censored (e.g., Heupel and Simpfendorfer 2002).

\section{Simulations}

\section{Methods}

We used the SURVIV program (White 1983) as modified by James Hines of Patuxent National Wildlife Research Center to do the simulations. The maximum likelihood method is used in SURVIV to estimate unknown parameters. For the tag return data, we assumed that 500 fish were tagged and released at the start of each year; for the telemetry data, our assumption was that 50 new fish were telemetered each year. We also assumed that surviving telemetered fish had functioning tags for the entire length of the study. The tag returns were tracked on a yearly basis, while the telemetry relocations were monthly. We used 500 replicate simulations for each run except when $p$ was less than 1 , in which case we used 1,000 .

We considered two different situations in the simulations: one in which the relocation probability for a telemetered fish was 1 and one in which it was less than 1 . When the relocation probability is 1 , we can explicitly calculate the number of fishing deaths in each period if we know the number of telemetered fish that were released at the beginning of each year, the number that are observed alive, and the number of natural deaths in each period. This makes the situation much simpler, and we can express the data in a single multinomial likelihood for each telemetered cohort. When $p$ is less than 1 , we can still use the likelihood equation (2) with two reasonable approximations: first, we can treat the number of animals relocated alive $\left(N_{j}\right)$ as fixed rather than conditional; and second, we can construct expressions for the expected number of live and dead fish first relocated on one to four search occasions after release (here we are assuming that for any reasonable values of $p$ fish are extremely unlikely to go five periods without being relocated).

\section{Results}

Case 1: certain relocation.-First we considered the case in which $F=0.5, M=0.2$, and $\lambda=0.6$ for 3 and 5 years of data. We obtained precise estimates of the annual $F \mathrm{~s}$ and the constant $M$ from the combination model, whether $\lambda$ was known or estimated (Table 2). The estimates from the combination model appeared to be essentially unbiased. The standard errors of the estimates in the combination model are smaller than those in the separate models (Tables 2, 3). A summary of the relative standard errors (RSEs, defined as SE/estimate) for the estimates of $M$ is given in Table 4. Our interpretation of these results is that the combination model (with $\lambda$ estimated) does somewhat better than the telemetry model in estimating $M$ and substantially better in estimating $F$. The combination model (with $\lambda$ known) does moderately better than the tag return model in estimating $F$ and substantially better in estimating $M$. It appears that the tag return and telemetry methods have different strengths and that the combination method takes advantage of the strengths of both.

We explored the relative contributions of telemetry and regular tags by running the same scenarios 
TABLE 2.-Average estimates of fishing mortality $(F)$, natural mortality $(M)$, and the tag reporting rate $(\lambda)$ and SEs for 3-year and 5-year combined models with annually varying values of $F$, a constant $M$, and true values as follows: $F=0.5, M=0.2$, and $\lambda=0.6$.

\begin{tabular}{|c|c|c|c|c|}
\hline \multirow{2}{*}{$\begin{array}{l}\text { Param- } \\
\text { eter }\end{array}$} & \multicolumn{2}{|c|}{$\lambda$ Known } & \multicolumn{2}{|c|}{$\lambda$ Estimated } \\
\hline & Estimate & SE & Estimate & SE \\
\hline \multicolumn{5}{|c|}{ Three-year combined model } \\
\hline$F_{1}$ & 0.50 & 0.0474 & 0.50 & 0.0580 \\
\hline$F_{2}$ & 0.50 & 0.0410 & 0.50 & 0.0557 \\
\hline$F_{3}$ & 0.51 & 0.0497 & 0.51 & 0.0691 \\
\hline$M$ & 0.20 & 0.0303 & 0.20 & 0.0330 \\
\hline$\lambda$ & 0.60 & & 0.60 & 0.0495 \\
\hline \multicolumn{5}{|c|}{ Five-year combined model } \\
\hline$F_{1}$ & 0.50 & 0.0480 & 0.50 & 0.0540 \\
\hline$F_{2}$ & 0.50 & 0.0396 & 0.50 & 0.0468 \\
\hline$F_{3}$ & 0.50 & 0.0384 & 0.50 & 0.0456 \\
\hline$F_{4}$ & 0.50 & 0.0396 & 0.50 & 0.0492 \\
\hline$F_{5}$ & 0.50 & 0.0396 & 0.50 & 0.0504 \\
\hline$M$ & 0.20 & 0.0180 & 0.20 & 0.0240 \\
\hline$\lambda$ & 0.60 & & 0.60 & 0.0345 \\
\hline
\end{tabular}

with 1,000 and 3,000 regular tags per year instead of 500. In the more realistic case of an unknown $\lambda$, we found that the RSEs of $M$ changed very little as we added regular tags. For the 3 years of data, the RSE went from 0.1645 with 500 regular tags per year to 0.1599 with 3,000 tags per year. For the 5 years of data, the RSE went from 0.1187 to 0.1113 . This shows clearly that the number of telemetry tags is very important in estimating $M$. To explore this further, we reran our scenarios with 25 telemetry tags per year instead of 50 (keeping the number of regular tags at 500 per year). The RSE of the $M$ estimate now increased markedly, from 0.1645 to 0.2327 for the 3 years of data and from 0.1187 to 0.1684 for the 5 years of data. However, we emphasize that the precision of our estimates is still quite reasonable even if we only use 25 telemetry tags per year.

A key advantage of the combination model is that the tag reporting rate $\lambda$ can be estimated reliably. The estimates are effectively unbiased, and the RSE was 0.0825 for the 3 years of data and 0.0575 for the 5 years of data. Rerunning the simulations with a much lower value of $\lambda(0.2)$, we found that the corresponding RSEs of the $\lambda$ estimates were 0.1119 and 0.0741 , respectively. We also note that in this case the tag return data alone would be really useless; the $M$ estimates had very little increase in RSE under the combined model ( 0.1260 versus 0.1187 for the 5 years of data).

In most of our simulations, we used scenarios in which we estimated annual $F$ s but assigned the true $F$ s so as to be equal every year. However, we
TABLE 3.-Average estimates of fishing mortality $(F)$, natural mortality $(M)$, and the tag reporting rate $(\lambda)$ and SEs for 3-year and 5-year tag return and telemetry models with annually varying values of $F$, a constant $M$, and true values as follows: $F=0.5, M=0.2$, and $\lambda=0.6$.

\begin{tabular}{|c|c|c|c|c|}
\hline \multirow{2}{*}{$\begin{array}{l}\text { Param- } \\
\text { eter }\end{array}$} & \multicolumn{2}{|c|}{ Tag return model } & \multicolumn{2}{|c|}{ Telemetry model } \\
\hline & Estimate & SE & Estimate & SE \\
\hline \multicolumn{5}{|c|}{ Three-year models } \\
\hline$F_{1}$ & 0.50 & 0.0546 & 0.51 & 0.1197 \\
\hline$F_{2}$ & 0.50 & 0.0525 & 0.51 & 0.0962 \\
\hline$F_{3}$ & 0.50 & 0.0663 & 0.51 & 0.0926 \\
\hline$M$ & 0.20 & 0.0608 & 0.20 & 0.0371 \\
\hline$\lambda$ & 0.60 & & & \\
\hline \multicolumn{5}{|c|}{ Five-year models } \\
\hline$F_{1}$ & 0.50 & 0.0535 & 0.51 & 0.1174 \\
\hline$F_{2}$ & 0.50 & 0.0443 & 0.51 & 0.0938 \\
\hline$F_{3}$ & 0.50 & 0.0444 & 0.50 & 0.0886 \\
\hline$F_{4}$ & 0.50 & 0.0456 & 0.50 & 0.0885 \\
\hline$F_{5}$ & 0.50 & 0.0486 & 0.50 & 0.0847 \\
\hline$M$ & 0.20 & 0.0264 & 0.20 & 0.0268 \\
\hline$\lambda$ & 0.60 & & & \\
\hline
\end{tabular}

also considered simulations in which the average value of $F$ was 0.5 but the value varied between years (we used $0.3,0.5$, and 0.7 for the 3 -year study and $0.3,0.4,0.5,0.6$, and 0.7 for the 5-year study). We found that the precision of the estimates of $M$ was essentially unchanged.

Estimates of the natural mortality rate varied in precision, depending on the level of fishing mortality and the approach used (tag return, telemetry, or combined). To explore this, we plotted the RSE of the $M$ estimates for $F$ ranging from 0.1 to 1 and $M$ ranging from 0.1 to 1 . For the tag return model, the estimates of $M$ became more precise as $F$ increased because the number of returned tags increased, particularly when $M$ was low (Figure 1). For the telemetry model, the estimates of $M$ became less precise as $F$ increased because fewer fish remained in the study so that fewer natural deaths were observed (Figure 2). The results for the combination model (Figures 3,4) were generally similar to those for the telemetry model be-

TABLE 4.-Comparison of relative SEs for natural mortality in 3-year and 5-year studies with annually varying values of fishing mortality and constant natural mortality.

\begin{tabular}{lcccccc}
\hline \multirow{2}{*}{ Model } & \multicolumn{2}{c}{ 3-Year study } & & \multicolumn{2}{c}{ 5-Year study } \\
\cline { 2 - 3 } \cline { 5 - 6 } \cline { 5 - 6 } & $\lambda$ Known & $\lambda$ Estimated & & $\lambda$ Known & $\lambda$ Estimated \\
\hline Combination & 0.1510 & 0.1645 & & 0.0903 & 0.1187 \\
Telemetry & & & 0.1852 & & & 0.1338 \\
Tag return & 0.3042 & & & 0.1319 & \\
\hline
\end{tabular}

a The telemetry tags do not involve the reporting rate of regular tags; the RSEs are included here for convenient comparison. 


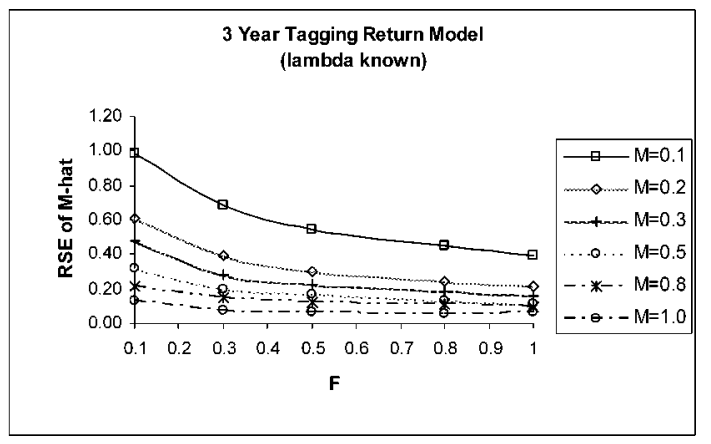

FIGURE 1.- Relative standard errors (RSEs) of estimates of natural mortality $(M)$ for different values of natural and fishing mortality $(F)$ in a 3-year tag return model with the tag reporting rate $(\lambda)$ assumed to be 0.6 .

cause the telemetry model is the dominant part of the combination structure. There is much more information in monthly telemetry locations than there is in yearly tag returns. Even if $F$ is low (say, 0.2 ), we can obtain precise estimates of $M$. Another advantage of the combination model is that we can obtain good estimates of $\lambda$. The best estimates of $\lambda$ are obtained when $F$ is high or $M$ is low because both situations result in the greatest number of tag returns (Figure 5).

The annual $F$ parameters can also be estimated reliably with the combined method. Based on the results in Table 2 for the 3-year study, the RSEs of the $F$ s are approximately $8-10 \%$ when $\lambda$ is known and approximately $11-14 \%$ when $\lambda$ is unknown. Note that the corresponding values for $M$ are 15 and $16 \%$, so that the fishing mortalities are estimated with slightly greater precision than the natural mortalities. Based on the results in Table

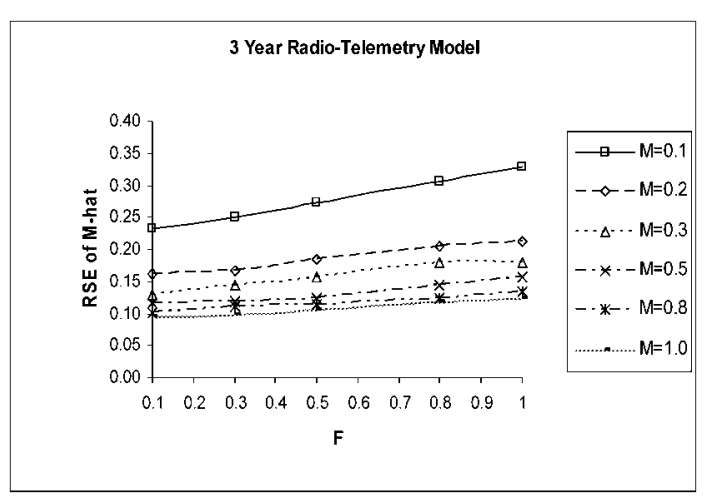

FIGURE 2.-Relative standard errors (RSEs) of estimates of natural mortality $(M)$ for different values of natural and fishing mortality $(F)$ in a 3-year telemetry tagging model.

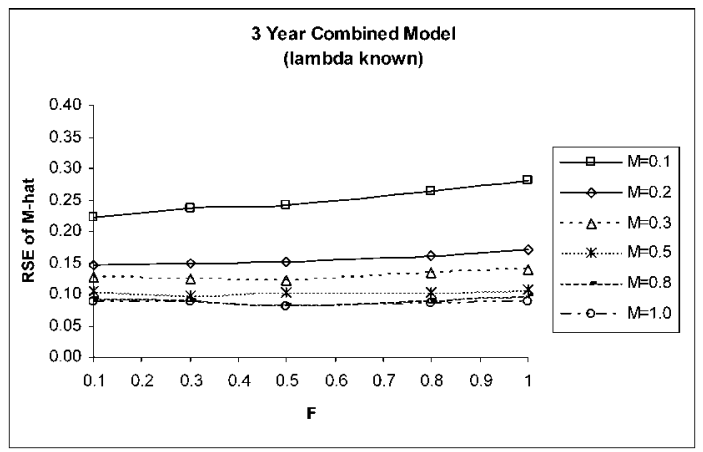

FIGURE 3.-Relative standard errors (RSEs) of estimates of natural mortality $(M)$ for different values of natural and fishing mortality $(F)$ in a 3-year combined tag return and telemetry model with the tag reporting rate $(\lambda)$ assumed to be 0.6 .

3 , the patterns are similar, although in this case there is little difference between the RSEs for the $F \mathrm{~s}$ and the $M \mathrm{~s}$. If $\lambda$ is known, the range is $8-10 \%$ for $F$ versus $9 \%$ for $M$; if $\lambda$ is unknown, the range is $9-11 \%$ for $F$ versus $12 \%$ for $M$. The precision is always higher for the $F \mathrm{~s}$ of the middle years than for those at the beginning or the end of the set of years studied.

Case 2: uncertain relocation.-We calculated the RSEs of the $F, M$, and $\lambda$ estimates in the combination model for different values of the relocation probability for a 3-year period and for the following sets of values: $(F=0.5, M=0.2),(F$ $=0.5, M=0.4),(F=0.2, M=0.2)$, and $(F=$ $0.2, M=0.4)$. As expected, there was a small penalty in terms of higher RSEs when the relocation rate was less than 1.0, but provided it was

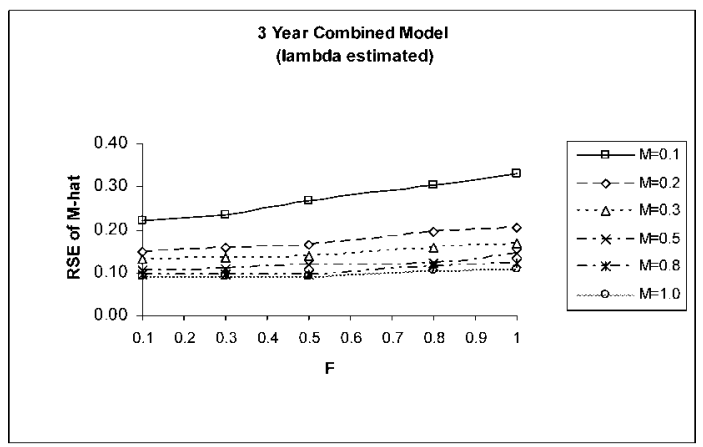

FIGURE 4.-Relative standard errors (RSEs) of estimates of natural mortality $(M)$ for different values of natural and fishing mortality $(F)$ in a 3-year combined tag return and telemetry model with the tag reporting rate $(\lambda)$ equal to 0.6 but assumed to be unknown and therefore estimated. 


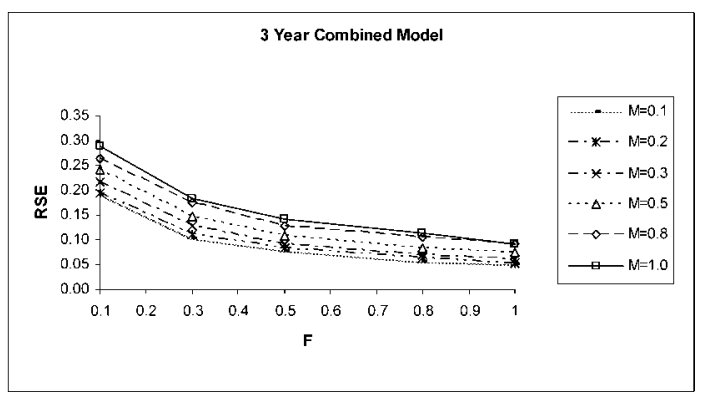

FIGURE 5.-Relative standard errors (RSEs) of estimates of the tag reporting rate $(\lambda)$ for different values of natural $(M)$ and fishing mortality $(F)$ in a 3-year combined tag return and telemetry model with $\lambda$ equal to 0.6 but assumed to be unknown and therefore estimated.

in the range $0.9-1.0$ the increases in the RSEs were no more than $10 \%$. For example, for the $(F=0.5$, $M=0.2$ ) case, we found that the RSE for the estimate of $M$ went from $18 \%$ to $20 \%$. The changes in the RSEs were even smaller for the estimates of $F$ and $\lambda$.

\section{Discussion}

Our results indicate that the combined model provides precise and essentially unbiased estimates of $F, M$, and $\lambda$. The strength of the tag return method is in estimating fishing mortality rates, whereas the telemetry method is better at estimating natural mortality rates. The combined method takes advantage of both and provides substantially better precision for estimates of $F$ and $M$ than either individual method. Further, even if $F$ is low, we can obtain precise estimates of $M$ unlike when we are using traditional tagging models. This is a big advantage in recovering fisheries, where tight regulation has kept the $F$ s low.

It is also possible to obtain precise estimates of the tag reporting rate with the combination method. This rate has been difficult to estimate in practice using a tag return model, even with multiyear data (Pollock et al. 1991; Hoenig et al. 1998a). Hearn et al. (1998) had to use pre- and postseason tagging methods to estimate the tag reporting rate. In our combination method, we can use typical once-a-year tagging data combined with the telemetry data to estimate the tag reporting rate as well as other parameters of interest.

We carried out additional simulations using longer multiyear data sets to estimate fishing and natural mortality rates and tag reporting rates from the tag return model alone. Even with 20 years of tagging data (annual releases of 500 tagged fish, with $F=0.5, M=0.2$, and $\lambda=0.6$ ), the $\lambda$ and
$M$ estimates were not at all reliable based on bias and precision. This agrees with the assessment of Hoenig et al. (1998a) that tag return data alone are not sufficient to estimate the reporting rate reliably. The question of how one can get reliable estimates of $\lambda$ and other parameters from a typical once-a-year tag return model is a very difficult one. This is a problem that deserves further investigation. We can see that our combination model offers a great advantage in obtaining reliable estimates of tag reporting rates (as well as fishing and natural mortality rates) using limited tag return and telemetry data.

Because of technological advances in telemetry equipment (Voegeli et al. 2001), researchers can now obtain detailed information about fish activity, movements, and rates and sources of mortality. For example, Heupel and Simpfendorfer (2002) used remote monitoring stations and acoustic transmitters to estimate the natural and fishing mortality of juvenile blacktip sharks Carcharhinus limbatus. A network of monitoring stations allowed the researchers to obtain essentially continuous information on movement and mortality.

Manual searching for telemetered fish can also be done to examine the timing and spatial patterns of natural and fishing mortality. For example, Hightower et al. (2001) conducted monthly searches for telemetered striped bass in a reservoir and found evidence of seasonal patterns in both fishing and natural mortality. Jepsen et al. (1998, 2000) carried out daily searches to estimate natural mortality rates and sources for radio-tagged Atlantic salmon Salmo salar and brown trout S. trutta smolts. Their studies illustrate that the patterns of movement indicating natural mortality can vary by species and size. Fish predation on a radio-tagged smolt was indicated by a decrease in transmitter signal strength (after the smolt was eaten by a northern pike Esox lucius or pikeperch [also known as zander] Sander lucioperca) or by tracking a transmitter into the shallow water typically occupied by northern pike. In some cases, predation was confirmed by electrofishing to capture predators with ingested transmitters. Predation by birds was established by tracking birds with ingested transmitters, locating a transmitter at an avian colony, or noting the disappearance of transmitters from the study area.

Although we have not explored it here, we believe that the combined method has another advantage that could be the subject of future research. We assumed that both methods were sampling the same population in an appropriate man- 
ner and focused on gaining precision in the estimates of fishing and natural mortality rates. Assuming that we had an external estimate of the tag reporting rate in a particular study, we could obtain estimates of fishing and natural mortality rates using tag returns alone, telemetry tags alone, or the two together. There may, however, be incompatibilities in the parameter estimates obtained with the various approaches. Therefore, having multiple incompatible estimates would be very useful in assessing the relative merits of the two types of data and which assumptions are most problematic.

\section{Acknowledgments}

We thank B. S. Brunhuber for editorial assistance. We thank James Hines of Patuxent Wildlife Research Center for help with writing and implementing SURVIV code.

\section{References}

Hearn, W. S., J. M. Hoenig, K. H. Pollock, and D. A. Hepworth. 2003. Tag-reporting rate estimation: III. Use of planted tags in one component of a multiplecomponent fishery. North American Journal of Fisheries Management 23:66-77.

Hearn, W. S., K. H. Pollock, and E. N. Brooks. 1998. Pre- and post-season tagging models and estimation of reporting rate and fishing and natural mortality rates. Canadian Journal of Fisheries and Aquatic Sciences 55:199-205.

Heupel, M. R., and C. A. Simpfendorfer. 2002. Estimation of mortality of juvenile blacktip sharks, Carcharhinus limbatus, within a nursery area using telemetry data. Canadian Journal of Fisheries and Aquatic Sciences 59:624-632.

Hightower, J. E., J. R. Jackson, and K. H. Pollock. 2001. Using telemetry methods to estimate natural and fishing mortality of striped bass in Lake Gaston, North Carolina. Transactions of the American Fisheries Society 130:557-567.

Hoenig, J. M., N. J. Barrowman, W. S. Hearn, and K. H. Pollock. 1998a. Multiyear tagging studies incorporating fishing effort data. Canadian Journal of Fisheries and Aquatic Sciences 55:1466-1476.

Hoenig, J. M., N. J. Barrowman, K. H. Pollock, E. N. Brooks, W. S. Hearn, and T. Polacheck. 1998b. Models for tagging data that allow for incomplete mixing of newly tagged animals. Canadian Journal of Fisheries and Aquatic Sciences 55:1477-1483.

Jepsen, N., K. Aarestrup, F. Okland, and G. Rasmussen. 1998. Survival of radio-tagged Atlantic salmon (Salmo salar L.) and trout (Salmo trutta L.) smolts passing a reservoir during seaward migration. Hydrobiologia 371/372:347-353.

Jepsen, N., S. Pedersen, and E. Thorstad. 2000. Behavioural interactions between prey (trout smolts) and predators (pike and pikeperch) in an impounded riv- er. Regulated Rivers: Research \& Management 16: 189-198.

Kaplan, E. L., and P. Meier. 1958. Nonparametric estimation from incomplete observations. Journal of the American Statistical Association 53:457-481.

Muoneke, M. I., and W. M. Childress. 1994. Hooking mortality: a review for recreational fisheries. Reviews in Fisheries Science 2:123-156.

Nasution, M., C. Brownie, and K. H. Pollock. 2002. Optimal allocation of sampling effort between regular banding and radio-tagging for estimating annual survival rates. Journal of Applied Statistics 29: 443-457.

Nasution, M., C. Brownie, K. H. Pollock, and R. E. Bennetts. 2001. Estimating survival from joint analysis of resighting and radiotelemetry capturerecapture data for wild animals. Journal of Agricultural, Biological, and Environmental Statistics 6: 461-478.

Nichols, J. D., S. L. Stokes, J. E. Hines, and M. J. Conroy. 1982. Additional comments on the assumption of homogeneous survival rates in modern band recovery estimation models. Journal of Wildlife Management 46:953-962.

Pascual, M. A., and O. O. Iribarne. 1993. How good are empirical predictions of natural mortality? Fisheries Research 16:17-24.

Pollock, K. H., C. M. Bunck, S. R. Winterstein, and C. L. Chen. 1995. A capture-recapture survival analysis model for radio-tagged animals. Journal of Applied Statistics 22:661-672.

Pollock, K. H., J. M. Hoenig, W. S. Hearn, and B. Calingaert. 2001. Tag reporting rate estimation: 1. An evaluation of the high-reward tagging method. North American Journal of Fisheries Management 21:521-532.

Pollock, K. H., J. M. Hoenig, W. S. Hearn, and B. Calingaert. 2002. Tag reporting rate estimation: 2. Use high reward tagging and observers in multi-component fisheries. North American Journal of Fisheries Management 22:727-736.

Pollock, K. H., J. M. Hoenig, and C. M. Jones. 1991. Estimation of fishing and natural mortality when a tagging study is combined with a creel survey or port sampling. Pages 423-434 in D. Guthrie, J. M. Hoenig, M. Holliday, C. M. Jones, M. J. Mills, S. A. Moberly, K. H. Pollock, and D. R. Talhelm, editors. Creel and angler surveys in fisheries management. American Fisheries Society, Symposium 12, Bethesda, Maryland.

Pollock, K. H., and D. G. Raveling. 1982. The assumptions of modern bird banding models, with emphasis on heterogeneous survival rates of population segments. Journal of Wildlife Management 46: 88-98.

Pollock, K. H., S. R. Winterstein, C. M. Bunck, and P. D. Curtis. 1989a. Survival analysis in telemetry studies: the staggered entry design. Journal of Wildlife Management 53:7-15.

Pollock, K. H., S. R. Winterstein, and M. J. Conroy. 1989b. Estimation and analysis of survival distributions for radio-tagged animals. Biometrics 45: 99-109. 
Powell, L. A., M. J. Conroy, J. E. Hines, J. D. Nichols, and D. G. Krementz. 2000. Simultaneous use of mark-recapture and radio telemetry to estimate survival, movement, and recapture rates. Journal of Wildlife Management 64:302-313.

Quinn, T. J., II, and R. B. Deriso. Quantitative fish dynamics. 1999. Oxford University Press, Oxford, UK.

Ricker, W. E. 1975. Computation and interpretation of biological statistics of fish populations. Fisheries Research Board of Canada Bulletin 191.

Seber, G. A. F. 1982. Estimation of animal abundance. MacMillan, New York.

Vetter, E. F. 1988. Estimation of natural mortality in fish stocks: a review. Fishery Bulletin 86:25-43.
Voegeli, F. A., M. J. Smale, D. M. Webber, Y. Andrade, and R. K. O'Dor. 2001. Ultrasonic telemetry, tracking, and automated monitoring technology for sharks. Environmental Biology of Fishes 60:267281.

White, G. C. 1983. Numerical estimation of survival rates from band recovery and biotelemetry data. Journal of Wildlife Management 47:717-728.

Wilde, G. R. 1998. Tournament-associated mortality in black bass. Fisheries 23(10):12-22.

Wilde, G. R., M. I. Muoneke, P. W. Bettoli, K. L. Nelson, and B. T. Hysmith. 2000. Bait and temperature effects on striped bass hooking mortality in freshwater. North American Journal of Fisheries Management 20:810-815. 